\title{
Performance Evaluation of the BZ COVID-19 Neutralizing Antibody Test for the Culture-Free and Rapid Detection of SARS-CoV-2 Neutralizing Antibodies
}

\author{
Bo Kyeung Jung ${ }^{1}\left(\mathbb{D}\right.$, Jung Yoon ${ }^{2, *}$, Joon-Yong Bae ${ }^{3}$, Jeonghun Kim ${ }^{3}$, Man-Seong Park ${ }^{3}$, Suk Yong Lee ${ }^{4}$ \\ and Chae Seung Lim ${ }^{2}(\mathbb{D}$ \\ 1 Department of Laboratory Medicine, Dankook University College of Medicine, Cheonan 31116, Korea; \\ lovegodmother@hanmail.net \\ 2 Department of Laboratory Medicine, College of Medicine, Korea University, Seoul 08308, Korea; \\ malarim@korea.ac.kr \\ 3 Department of Microbiology, Institute for Viral Diseases, College of Medicine, Korea University, \\ Seoul 02841, Korea; harbe3103@korea.ac.kr (J.-Y.B.); totspurs@daum.net (J.K.); ms0392@korea.ac.kr (M.-S.P.) \\ 4 Creativity Lab., Creativity \& Innovation Center, Samsung Electronics, Suwon 16677, Korea; \\ syren.lee@diavision.co.kr \\ * Correspondence: unoaotro@korea.ac.kr
}

check for

updates

Citation: Jung, B.K.; Yoon, J.; Bae, J.-Y.; Kim, J.; Park, M.-S.; Lee, S.Y.; Lim, C.S. Performance Evaluation of the BZ COVID-19 Neutralizing Antibody Test for the Culture-Free and Rapid Detection of SARS-CoV-2 Neutralizing Antibodies. Diagnostics 2021, 11, 2193. https://doi.org/ 10.3390/diagnostics11122193

Academic Editor: Alessandro Russo

Received: 27 October 2021

Accepted: 23 November 2021

Published: 25 November 2021

Publisher's Note: MDPI stays neutral with regard to jurisdictional claims in published maps and institutional affiliations.

Copyright: (C) 2021 by the authors. Licensee MDPI, Basel, Switzerland. This article is an open access article distributed under the terms and conditions of the Creative Commons Attribution (CC BY) license (https:/ / creativecommons.org/licenses/by/ $4.0 /)$.

\begin{abstract}
Rapid and accurate measurement of SARS-CoV-2 neutralizing antibodies (nAbs) can aid in understanding the development of immunity against COVID-19. This study evaluated the diagnostic performance of a rapid SARS-CoV-2 nAb detection test called the BZ COVID-19 nAb test BZ-nAb (BZ-nAb; BioZentech). Using the 90\% plaque-reduction neutralization test (PRNT-90) as a reference, 104 serum specimens collected from COVID-19-positive and -negative patients were grouped into 40 PRNT-90-positive and 64 PRNT-90-negative specimens. The performance of the BZ-nAb was compared with that of the cPass surrogate virus neutralization test (cPass sVNT; Genscript). The BZ-nAb showed a sensitivity ranging from $92.5 \%-95.0 \%$ and specificity ranging from $96.9 \%-100 \%$, whereas cPass sVNT showed a sensitivity of $100 \%$ (95\% confidence interval (CI) $90.5 \%-100 \%$ ) and specificity of $98.4 \%$ (95\% CI, 91.6\%-100\%). The dilution factor obtained with PRNT-90 showed a stronger correlation with the percent inhibition of cPass sVNT ( $\mathrm{r}=0.8660, p<0.001)$ compared with the test and control line ratio $(\mathrm{T} / \mathrm{C}$ ratio) of the BZ-nAb $(\mathrm{r}=-0.7089, p<0.001)$. An almost perfect agreement was seen between the $\mathrm{BZ}-\mathrm{nAb}$ and $\mathrm{cPass}$ sVNT results, with a strong negative correlation between the BZ-nAb T/C ratio and cPass sVNT percent inhibition $(\mathrm{r}=-0.8022, p<0.001)$. In conclusion, the diagnostic performance of the BZ-nAb was comparable to that of the cPass sVNT, although the BZ-nAb had a slightly lower sensitivity.
\end{abstract}

Keywords: SARS-CoV-2; COVID-19; neutralizing antibodies; RDT; ELISA

\section{Introduction}

Coronavirus disease 19 (COVID-19), caused by severe acute respiratory syndrome coronavirus 2 (SARS-CoV-2), has been global pandemic disease since March of 2020. Immunity against SARS-CoV-2, induced either by infection or vaccination, has been shown to protect against subsequent reinfection and/or reduce the risk of developing a severe form of the disease [1,2]. Previous studies have shown that neutralizing antibodies (nAbs) against the receptor-binding domain (RBD) of the spike (S) glycoprotein of SARS-CoV-2 provide protective immunity against COVID-19 [3-5]. The nAb response against SARS-CoV-2 has been shown to be associated with patient survival, and the titer value is predictive of the extent of immunity developed by the host [6,7]. With the introduction of multiple SARS-CoV-2 vaccines, development of the $\mathrm{nAb}$ response and neutralization titers have been used to quantify the immunogenicity of vaccines in clinical trials [8-10]. 
nAb-testing assays conventionally include live-cell neutralization tests, such as the plaque reduction neutralization test (PRNT) and microneutralization assay. Traditionally, both PRNT and the microneutralization assay have been used to quantify the nAb response; however, the PRNT has been found to be more sensitive than the microneutralization assay for measuring SARS-CoV-2-specific nAbs [11,12]. These live-cell neutralization tests are limited by the need to be performed in a biosafety level (BSL) 3 laboratory and their small throughput, in addition to the high cost and skill required to perform them. A pseudovirus neutralization test using a pseudovirus expressing the SARS-CoV-2specific S glycoprotein [13] can be performed in a BSL-2 facility; however, it requires the maintenance of virus cultures, which is not feasible in terms of the required facilities, cost, and resultant throughput.

A blocking ELISA called the cPass surrogate virus neutralization test (sVNT) (GenScript, Piscataway, NJ, USA) is also commercially available. cPass sVNT is designed to specifically measure the levels of nAbs against the RBD of the S glycoprotein of SARS$\mathrm{CoV}-2$ and assesses the neutralizing capacity of nAbs, enabling the detection of functional SARS-CoV-2 neutralization activity [14]. The cPass sVNT results showed high agreement with those of live-cell neutralization detection methods [14-16]. In contrast to live-cell neutralization tests, cPass sVNT is performed without the need for virus cultures and requires only a few hours to complete. However, since cPass sVNT is an ELISA, specialized equipment for optical density measurements is still required.

Recently, rapid diagnostic tests (RDTs) for SARS-CoV-2 nAb detection, such as the BZ COVID-19 nAb test (BZ-nAb; BioZentech, Seoul, Korea), have been developed. The $\mathrm{BZ}-\mathrm{nAb}$ is an immunochromatographic assay involving two components: the purified RBD of $S$ protein and the angiotensin-converting enzyme 2 (ACE2) receptor. Similar to the cPass sVNT, the BZ-nAb also detects SARS-CoV-2 nAbs by detecting the degree of inhibition of the RBD-ACE2 interaction by nAbs in the sample. Theoretically, any immunoglobulin isotype targeting the RBD can be detected by the BZ-nAb. The BZ-nAb can be performed within 10-15 $\mathrm{min}$, and the test results are obtained by the visual interpretation of the test line [17].

The purpose of this study was to evaluate the performance of the BZ-nAb to detect SARS-CoV-2 nAbs in serum samples of COVID-19-positive and -negative patients. The performance of the BZ-nAb was compared with that of cPass sVNT, which was used as the reference test for SARS-CoV-2 nAb detection. In addition to visual interpretation, the signal intensity of each line (both test and control) was analyzed using a smartphone-based image analysis application (SIA). With the development of mobile medical applications for automated interpretation of RDT results, analyzing RDTs with these applications has been reported to provide improved and reliable results [18-20]. A pilot study of SIA evaluation using different immunochromatographic assay and results showed acceptable performance (100\% sensitivity and $97.8 \%$ specificity) when compared to visual interpretation results (data not shown). Our performance evaluation of the BZ-nAb involved the evaluation of two kinds of results, one obtained by visual interpretation, and the other measured by the SIA. The correlation between the results of the BZ-nAb and those of cPass sVNT was also determined.

\section{Materials and Methods}

\subsection{Sample Collection and Preparation}

Serum samples from COVID-19-positive and -negative patients were collected from the Korea University Guro Hospital from March 2020 to December 2020. Patients were confirmed to be COVID-19-positive using real-time polymerase chain reaction (RT-PCR)mediated detection of SARS-CoV-2. Patients with no reported history of COVID-19, were not vaccinated against SARS-CoV-2, and confirmed negative upon RT-PCR testing were designated COVID-19-negative patients. A total of 104 samples (45 COVID-19-positive samples and 59 COVID-19-negative samples) were collected from 100 patients (47 males and 53 females). Two samples with different intervals from the onset of symptoms were 
collected from four COVID-19 patients. The median age of patients was 63 years (ranged from 17 to 88 years). For COVID-19-positive patients, the median time after symptom onset was 17 days (ranged from 0 to 66 days). The presence of $n A b$ against SARS-CoV-2 was confirmed using the PRNT, which was the reference method in this study, and serum samples were grouped according to these results. Serum samples were stored at $-80{ }^{\circ} \mathrm{C}$ until analysis. Three samples out of a total of 104 samples were not subjected to cPass sVNT due to insufficient sample volume. This study was approved by the Institutional Review Board of the Korea University Guro Hospital (2021GR0146).

\subsection{PRNT}

The PRNT was performed as described previously [21]. Briefly, serum samples were diluted 1:10 using phosphate-buffered saline and serially diluted by two-fold to 1:160. A total of $100 \mu \mathrm{L}$ of diluted serum was mixed with an equal volume of approximately 100 plaque-forming units of SARS-CoV-2 (BetaCoV/Korea/KCDC03/2020, NCCP 43326), resulting in final titers ranging from 1:20 to 1:320, and incubated for $1 \mathrm{~h}$ at $37^{\circ} \mathrm{C}$ and $5 \%$ $\mathrm{CO}_{2}$. Vero cell plates (NEST Scientific, SPL Life Science, Pochen, Korea) were inoculated with the serum-virus mixtures, incubated for $1 \mathrm{~h}$ at $37^{\circ} \mathrm{C}$ and $5 \% \mathrm{CO}_{2}$ and overlaid with agar for $72 \mathrm{~h}$ at $37{ }^{\circ} \mathrm{C}$ and $5 \% \mathrm{CO}_{2}$. Then, the plates were stained using $0.5 \%$ crystal violet, and the number of plaques was enumerated. A $90 \%$ plaque-reduction neutralization (PRNT-90) was the maximum dilution that resulted in a 90\% reduction in plaques when compared with that of control plates. Samples with PRNT-90 titer $\geq 1: 20$ was considered PRNT-90-positive, and the remaining were considered PRNT-90-negative.

\section{3. cPass sVNT}

The cPass sVNT (GenScript, Piscataway, NJ, USA) was performed according to the manufacturer's instructions. Serum samples were diluted 1:10 with the sample dilution buffer provided in the kit. The diluted samples were mixed with an equal volume of horseradish peroxidase-conjugated recombinant SARS-CoV-2 RBD fragment solution diluted with RBD dilution buffer. Next, $100 \mu \mathrm{L}$ of this solution was added to the 96-well plate coated with the human ACE2 receptor and incubated for $30 \mathrm{~min}$ at $37^{\circ} \mathrm{C}$. The plate was washed four times with the provided wash solution. Then, 3,3',5,5'-tetra-methylbenzidine was added to each well and incubated for $15 \mathrm{~min}$ in the dark, followed by addition of the stop solution. Optical density at $450 \mathrm{~nm}$ was measured and compared to that of the control wells. Percentage signal inhibition was calculated, and a cutoff $\geq 30 \%$ was considered positive, according to the manufacturer's instructions.

\section{4. $B Z-n A b$}

The BZ-nAb is an immunochromatographic assay involving two components: the Gold conjugated SARS-CoV-2 S protein RBD and the ACE2 receptor. For internal control, Gold conjugated Chicken IgY and goat anti-chicken IgY are included (Figure 1A). The BZ-nAb detects SARS-CoV-2 nAbs by detecting the inhibition of the RBD-ACE2 interaction by $n A b s$ in the sample (Figure 1B,C).

A total of $40 \mu \mathrm{L}$ of serum and $90 \mu \mathrm{L}$ of RBD-containing buffer was added to the specimen well of the BZ-nAb cassette (BioZentech, Seoul, Korea). After 13-15 min of incubation, the intensities of the control and test lines were visually compared, and a sample was considered positive as follows: (1) absence of the test line with presence of the control line. (2) The intensity of test line lower than that of the control line (Figure S1). The line intensity is inversely proportional to the $\mathrm{nAb}$ titer.

The signal intensities of BZ-nAb were also analyzed using an SIA (Figure S1). Immediately after the visual interpretation of BZ-nAb, images were captured using a smartphone camera (Galaxy S20 plus, Samsung), and the intensity for both the test and control lines was analyzed by the SIA. The exposure of the light was designed to be constant using the smartphone flash software algorithm. Images were captured only when the shooting focus was within the proper measuring distance, which was provided on the screen as the 
guidelines. All images were converted to color spectrum data by each color, and the pixel intensities of test line were calculated percentiles compared with control line intensities by same the color spectrum data. The mean pixel intensity of the test and control lines was determined and used to calculate the percent test to control pixel intensity ratio ( $\mathrm{T} / \mathrm{C}$ ratio) as follows: $\mathrm{T} / \mathrm{C}$ ratio $(\%)=$ (test line pixel intensity $/$ control line pixel intensity) $\times 100 \%$.

\subsection{Statistical Analysis}

The $\mathrm{T} / \mathrm{C}$ ratios of the $\mathrm{BZ}-\mathrm{nAb}$ were analyzed using receiver operating characteristic (ROC) analysis to select the optimal cutoff consistent with the visual interpretation results. The $95 \%$ confidence interval for sensitivity and specificity of the BZ-nAb and the cPass sVNT using PRNT-90 as the reference, were compared to evaluate their performance for the detection of SARS-CoV-2 nAbs. Agreement of the BZ-nAb and cPass sVNT results were verified using Cohen's kappa coefficient $(\mathrm{K})$, wherein the strength of agreement was defined as follows: $\mathrm{K}<0$, poor; $0-0.2$, slight; $0.21-0.4$, fair; $0.41-0.6$, moderate; $0.61-0.8$, substantial; and $0.81-1$, almost perfect. The correlations between the values obtained from aforementioned three methods were analyzed using Pearson's correlation analysis. Differences with $p$-values $<0.05$ were considered statistically significant. Statistical analyses were performed using MedCalc version 18.11.6 (MedCalc Software bvba, Ostend, Belgium), and visualizations were performed using MedCalc and R software (version 3.4.3).

A Schematic diagram of BZ-nAb kit

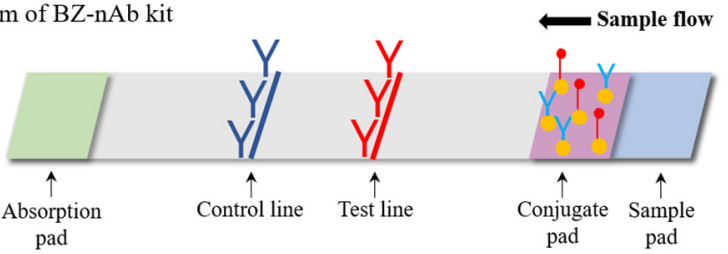

B Positive sample
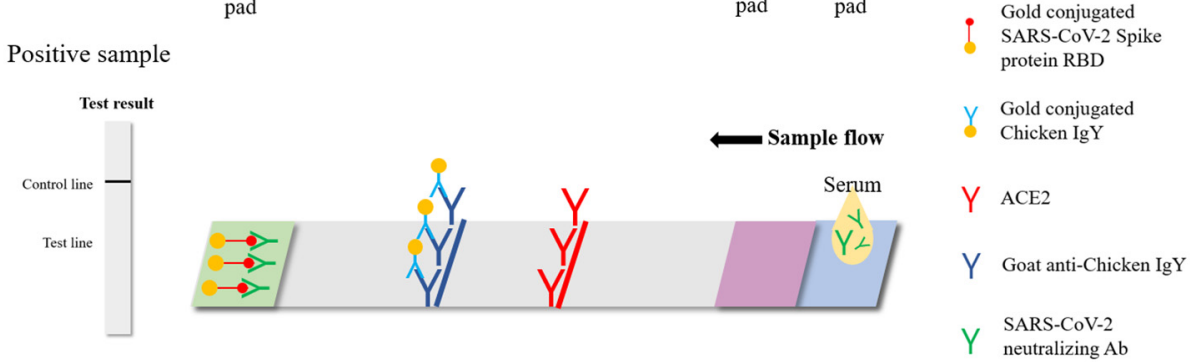

C Negative sample
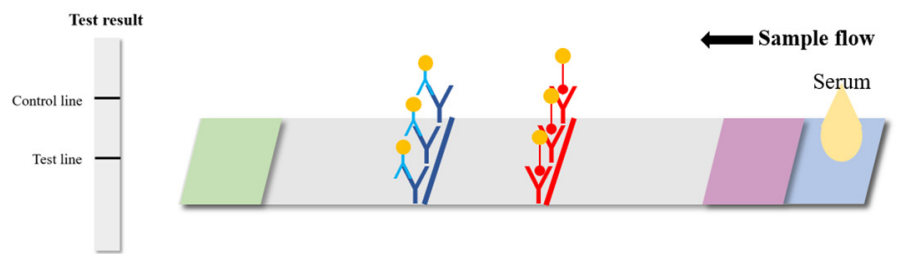

Figure 1. BZ-nAb test kit. (A) Schematic diagram of the BZ-nAb is shown. The diagram of SARS$\mathrm{CoV}-2$ neutralizing antibody positive (B) and negative (C) samples. In SARS-CoV-2 nAb positive samples, the RBD-ACE2 interaction in the test line are inhibited by nAbs in the sample, and vice versa in the negative sample.

\section{Results}

A total of 104 samples (45 COVID-19-positive samples and 59 COVID-19-negative samples) were used in this study. PRNT-90 was used as the reference for the detection of SARS-CoV-2 nAbs, and the samples were grouped based on the obtained results. Of the 45 COVID-19 samples, six samples showed negative PRNT-90 results and were, thus, included in the PRNT-90-negative group. Of the six samples with negative PRNT-90 results, five samples were collected less than 5 days after the symptom onset ( 2 samples on day 0 , 1 sample on day 2 , and 2 samples on day 4), and one sample was collected 10 days after the 
symptom onset. Of the 59 presumed COVID-19 negative samples, one sample showed a positive PRNT-90 result (1:40) and was included in the PRNT-90-positive group. In total, 40 and 64 samples were included in the PRNT-90-positive and -negative groups, respectively. The distribution of dilution factors for the PRNT-90-positive and -negative groups is shown in Figure 2A.
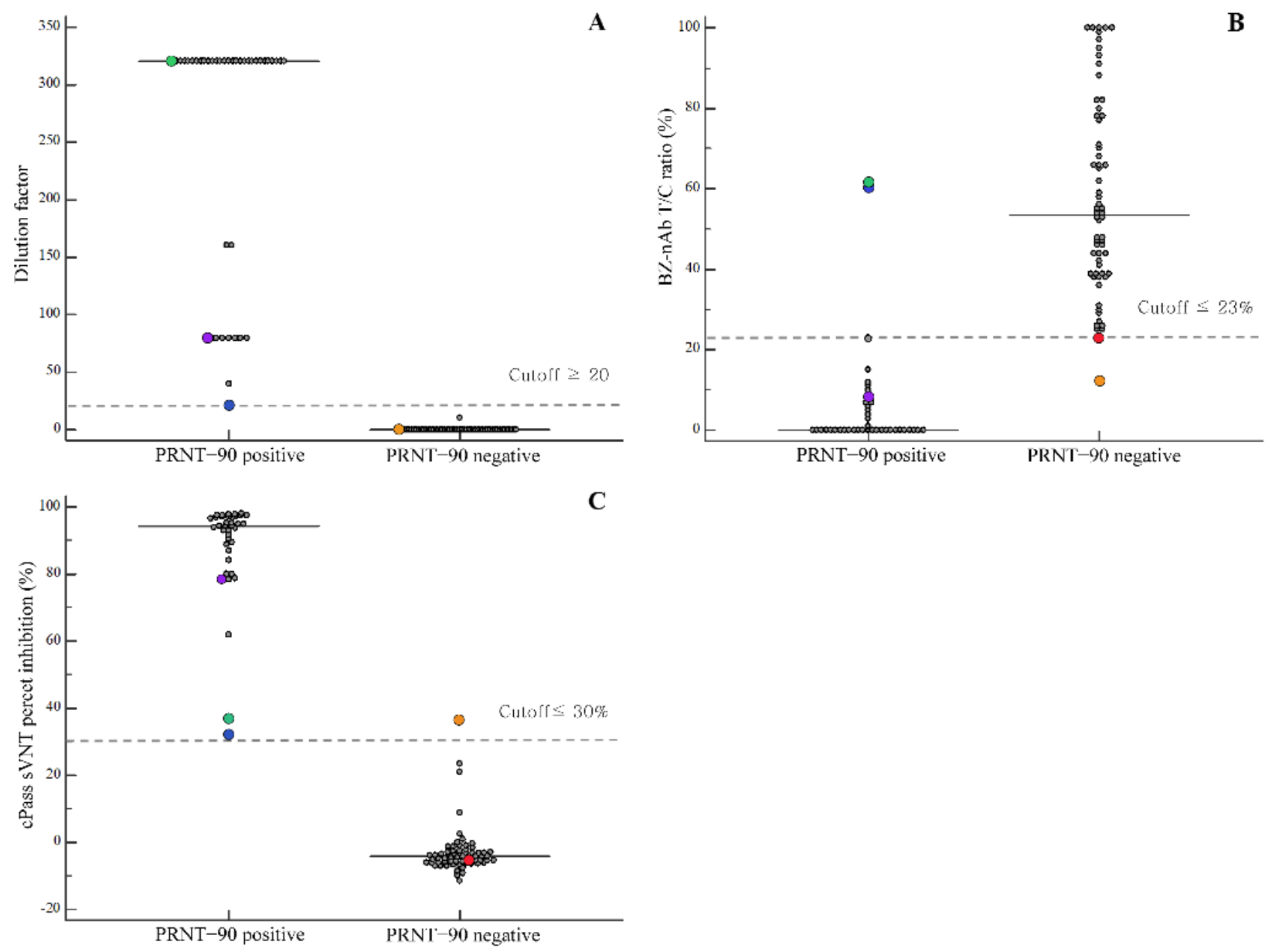

Figure 2. Data distribution and comparison between PRNT-90-positive and -negative groups. (A) PRNT-90 dilution factor; (B) BZ-nAb T/C ratio; (C) cPass sVNT percent inhibition. When compared with the PRNT-90 positive samples, the BZ-nAb showed three discordant results when visually interpreted (green, blue, purple dots), and two when interpreted using $\mathrm{T} / \mathrm{C}$ ratio (green, blue dots). When compared with the PRNT-90 negative samples, the BZ-nAb showed two discordant results using $\mathrm{T} / \mathrm{C}$ ratio (red, orange dots), and one discordant result was also noted with cPass sVNT (orange dot). The horizontal dashed lines indicate the cutoffs for each assay.

\subsection{BZ-nAb Test Line Intensity Measurement by the SIA}

For all samples subjected to the BZ-nAb, the intensities of the test and control lines were measured using an SIA, and the $\mathrm{T} / \mathrm{C}$ ratio was calculated. The median $\mathrm{T} / \mathrm{C}$ ratio for the visually interpreted BZ-nAb positive and negative groups were $0 \%$ and $54.0 \%$, respectively (Figure $3 \mathrm{~A}$ ). The 90 th percentile $\mathrm{T} / \mathrm{C}$ ratio for the $\mathrm{BZ}-\mathrm{nAb}$ visually interpreted positive and negative groups was $13.5 \%$ and $97.2 \%$, respectively. ROC analysis of the T/C ratio showed that a cutoff equal to or lower than $23 \%$ were visually interpreted positive BZ-nAb samples with a sensitivity of $100 \%$ and a specificity of $95.5 \%$ (area under curve 0.997) (Figure 3B). 
$\mathbf{A}$

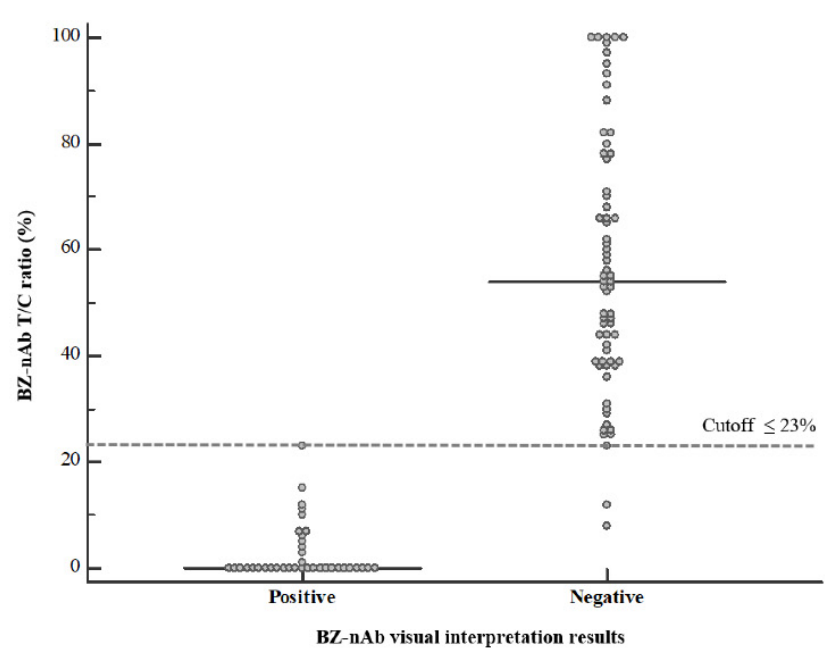

B

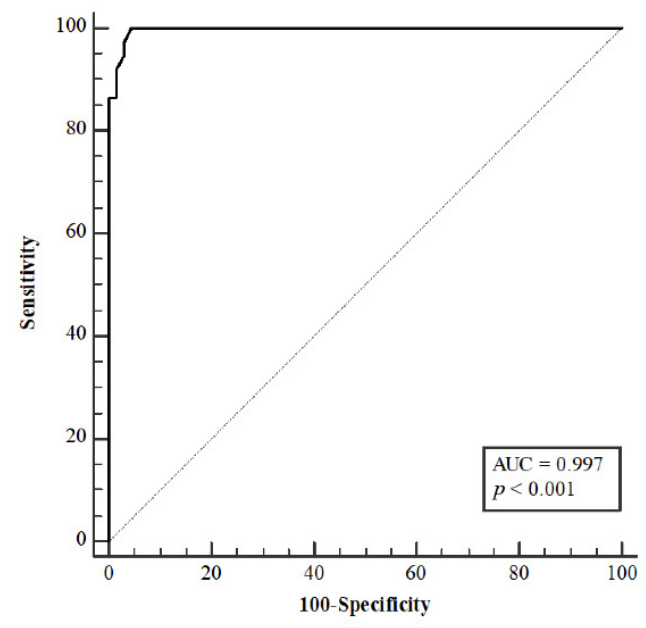

Figure 3. Comparison of the T/C ratio-interpreted and visually interpreted results of the BZ-nAb test. (A) Data distribution of BZ-nAb visually interpreted results. The horizontal dashed line indicates the cutoff selected using the receiver operating characteristic (ROC) analysis. (B) The ROC curve for the T/C ratio results, compared to the visually interpreted results. The area under the curve (AUC) values are also presented.

\subsection{Performance Evaluation of the BZ- $n A b$ and cPass $s V N T$ for the Detection of $S A R S-C o V-2 n A b s$}

The estimated diagnostic performances of BZ-nAb and cPass sVNT are shown in Table 1. For the BZ-nAb, the effect of the $T / C$ ratio cutoff obtained by ROC analysis (cutoff $\leq 23 \%$ ) was also analyzed. The BZ-nAb showed a sensitivity ranging from $92.5 \%$ to $95.0 \%$ and a specificity of $96.9 \%$ to $100 \%$. Interpretation using the $\mathrm{T} / \mathrm{C}$ ratio increased BZ-nAb sensitivity but lowered specificity. The cPass sVNT was the more sensitive method compared to the BZ-nAb, regardless of the interpretation method. However, the specificity of the cPass sVNT was lower than that of the BZ-nAb when results were visually interpreted (Table 1).

Table 1. Performance comparison of the BZ COVID-19 neutralizing antibody test and cPass surrogate viral neutralization assay.

\begin{tabular}{|c|c|c|c|c|c|c|c|c|}
\hline \multirow{2}{*}{$\begin{array}{l}\text { Neutralizing Ab } \\
\text { Detection Methods }\end{array}$} & \multicolumn{2}{|c|}{ Total Number of Samples } & \multirow{2}{*}{$\begin{array}{c}\text { True } \\
\text { Positive }\end{array}$} & \multirow{2}{*}{$\begin{array}{l}\text { False } \\
\text { Positive }\end{array}$} & \multirow{2}{*}{$\begin{array}{c}\text { False } \\
\text { Negative }\end{array}$} & \multirow{2}{*}{$\begin{array}{c}\text { True } \\
\text { Negative }\end{array}$} & \multirow{2}{*}{$\begin{array}{c}\text { Sensitivity }^{a} \\
(95 \% \text { CI })\end{array}$} & \multirow{2}{*}{$\begin{array}{c}\text { Specificity } \\
(95 \% \text { CI })\end{array}$} \\
\hline & $\begin{array}{l}\text { PRNT-90 } \\
\text { Positive }\end{array}$ & $\begin{array}{l}\text { PRNT-90 } \\
\text { Negative }\end{array}$ & & & & & & \\
\hline \multicolumn{9}{|l|}{$\begin{array}{c}\text { BZ COVID-19 } \\
\text { neutralizing Ab test }\end{array}$} \\
\hline Visual interpretation & 40 & 64 & 37 & 0 & 3 & 64 & $\begin{array}{c}92.5 \\
(79.6-98.4)\end{array}$ & $\begin{array}{c}100 \\
(94.4-100)\end{array}$ \\
\hline $\begin{array}{l}\text { Interpretation using } \\
\mathrm{T} / \mathrm{C} \text { ratio cutoff }\end{array}$ & 40 & 64 & 38 & 2 & 2 & 62 & $\begin{array}{c}95.0 \\
(83.1-99.4)\end{array}$ & $\begin{array}{c}96.9 \\
(89.2-99.6)\end{array}$ \\
\hline $\begin{array}{l}\text { cPass surrogate virus } \\
\text { neutralization test }\end{array}$ & 37 & 64 & 37 & 1 & 0 & 63 & $\begin{array}{c}100 \\
(90.5-100)\end{array}$ & $\begin{array}{c}98.4 \\
(91.6-100)\end{array}$ \\
\hline
\end{tabular}

$\mathrm{Ab}$, antibody; $\mathrm{CI}$, confidence interval; PRNT-90, 90\% plaque reduction neutralization test; $\mathrm{T} / \mathrm{C}$ ratio, test to control pixel intensity ratio.

a $($ True positive/(True positive + False negative) $) \times 100$ using PRNT-90 as the reference. ${ }^{b}$ (True negative/(True negative + False positive))

$\times 100$ using PRNT-90 as the reference.

Comparisons between PRNT-90-positive and -negative groups with respect to the distributions of dilution factor for PRNT-90, T/C ratio for the BZ-nAb, and percent inhibition for the cPass sVNT are shown in Figure 2. For PRNT-90-positive and -negative groups, the median $\mathrm{T} / \mathrm{C}$ ratios were $0 \%$ and $53.5 \%$, and the median percentage inhibition was $94.2 \%$ and $-4.2 \%$, respectively. Among the PRNT-90-positive samples, three samples were false-negatives according to the results of visual interpretation of BZ-nAb (Figure 2 
and Figure S2A, green, blue, and purple dots), and two samples were also false-negatives when interpreted using the T/C ratio cutoff (Figure 2 and Figure S2A, blue and green dots). These two false-negative samples showed PRNT-90 titers of 1:20 and above 1:320 and were positive for cPass sVNT with relatively low cPass sVNT percentage inhibition values (31.2\% and $36.1 \%$, respectively). One PRNT-90 negative sample was a false-positive according to both the BZ-nAb using $\mathrm{T} / \mathrm{C}$ ratio cutoff and the cPass sVNT; however, this sample was negative according the BZ-nAb visually interpreted result (Figure 2 and Figure S2B, orange dot). The dilution factor of PRNT-90 was more strongly correlated with cPass sVNT percent inhibition than with the $\mathrm{T} / \mathrm{C}$ ratio of the $\mathrm{BZ}-\mathrm{nAb}(\mathrm{r}=0.8660$ vs. $\mathrm{r}=-0.7089$; both $p<0.001$ ).

\subsection{Agreement of BZ-nAb and cPass sVNT Results}

An almost perfect agreement was observed between the results of cPass sVNT and BZ$\mathrm{nAb}$ with visual interpretation ( $\kappa=0.914,95 \%$ confidence interval $(\mathrm{CI}): 0.831-0.996)$ and that of the cPass sVNT and BZ-nAb with T/C ratio interpretation ( $k=0.936,95 \% \mathrm{CI}$ : $0.866-1)$. The results of the $\mathrm{BZ}-\mathrm{nAb}$ with $\mathrm{T} / \mathrm{C}$ ratio interpretation showed a higher positive agreement, but lower negative agreement, with cPass sVNT results compared to the results of the BZ-nAb test obtained by visual interpretation (Table 2). There was a strong negative correlation between BZ-nAb T/C ratio and cPass sVNT percent inhibition $(\mathrm{r}=-0.8022$, $p<0.001$ ) (Figure 4).

Table 2. Agreement between BZ COVID-19 neutralizing antibody test and cPass surrogate viral neutralization assay.

\begin{tabular}{|c|c|c|c|c|c|c|c|}
\hline & & \multicolumn{2}{|c|}{ cPass sVNT Results } & \multicolumn{3}{|c|}{ Percent Agreement $\left(95 \%\right.$ CI) ${ }^{a}$} & \multirow{2}{*}{$\begin{array}{c}\text { Kappa } \\
\text { Statistic } \\
(95 \% \text { CI })\end{array}$} \\
\hline & & Positive & Negative & Positive & Negative & Overall & \\
\hline BZ-nAb with visual & Positive & 34 & 0 & 89.5 & 100 & 96.0 & 0.914 \\
\hline interpretation & Negative & 4 & 63 & $(75.2-97.1)$ & $(94.3-100)$ & $(90.2-98.9)$ & $(0.831-0.996)$ \\
\hline \multirow{2}{*}{$\mathrm{BZ}-\mathrm{nAb}$ with $\mathrm{T} / \mathrm{C}$ ratio cutoff } & Positive & 36 & 1 & 94.7 & 98.4 & 97.0 & 0.936 \\
\hline & Negative & 2 & 62 & $(82.3-99.4)$ & $(91.5-100)$ & $(91.6-99.4)$ & $(0.866-1)$ \\
\hline
\end{tabular}

BZ-nAb, BZ COVID-19 neutralizing antibody test; CI, confidence interval; cPass sVNT, cPass surrogate viral neutralization assay; T/C ratio, test to control the pixel intensity ratio. ${ }^{a}$ Positive agreement was calculated (BZ-nAb and cPass sVNT positive/cPass sVNT positive) $\times 100$, negative agreement was calculated (BZ-nAb and cPass sVNT negative/cPass sVNT negative) $\times 100$, and overall agreement was calculated (positive or negative by both BZ-nAb and cPass sVNT/(cPass sVNT positive $+\mathrm{cPass}$ sVNT negative) $\times 100$.

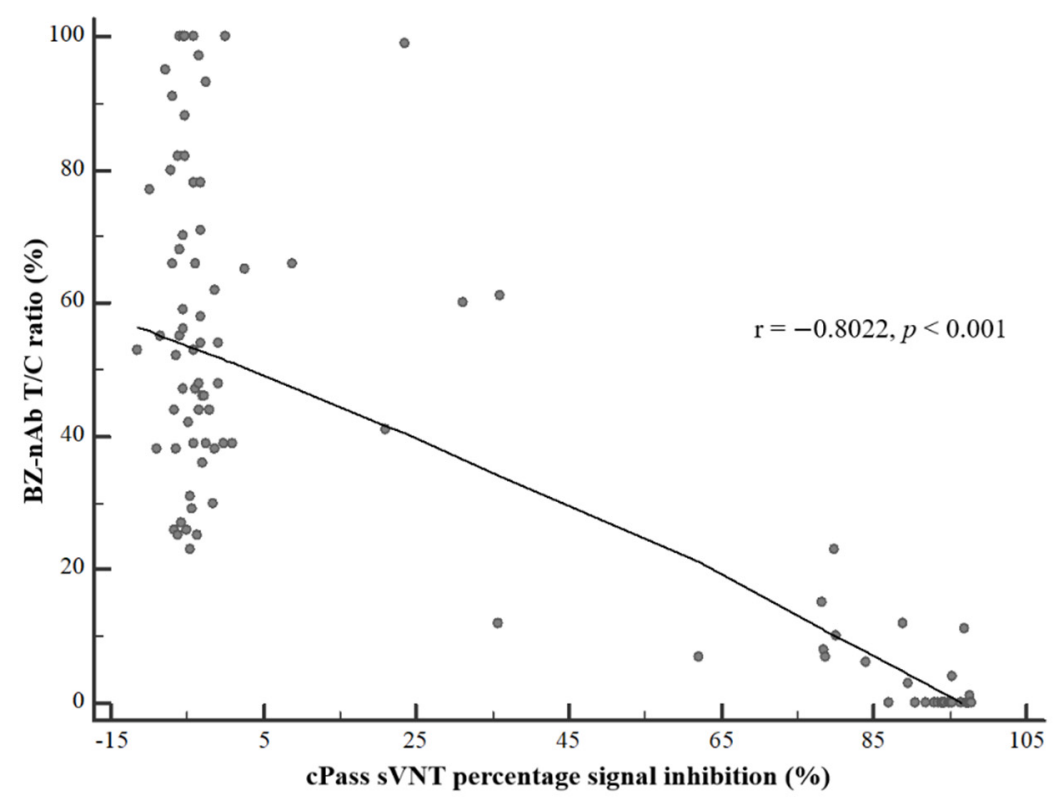

Figure 4. Correlation between the BZ-nAb T/C ratio and cPass sVNT percent inhibition. Pearson's correlation analysis showed a strong negative correlation between BZ-nAb T/C ratio and cPass sVNT percent inhibition $(r=-0.8022, p<0.001)$ when analyzed using a total of 101 samples. 


\section{Discussion}

Rapid and accurate SARS-CoV-2-specific nAb measurement can provide additional insight into understanding the development of host immunity against COVID-19 and vaccine development and aid in characterizing convalescent plasma for therapy. To the best of our knowledge, the BZ-nAb is the first RDT designed to detect SARS-CoV-2 nAbs based on the neutralizing capacity of nAbs. RDTs can be rapidly and easily performed, contributing towards its feasibility as standard SARS-CoV-2 $\mathrm{nAb}$ detection tests; however, they show varying degrees performance. In this study, we evaluated the diagnostic performance of the BZ-nAb by comparing it to that of the cPass sVNT, using the PRNT-90 as a reference. In terms of SARS-CoV-2 nAb detection, the BZ-nAb showed a diagnostic performance comparable to that of the cPass sVNT, although the sensitivity of the BZ-nAb was slightly lower than that of the cPass sVNT.

The overall performance of the BZ-nAb was acceptable, regardless of the interpretation method. Although the correlation between dilution factor of PRNT-90 and T/C ratio of $\mathrm{BZ}-\mathrm{nAb}$ was strong, the impact of dilution factors on the false negative samples was not definite. The two false negative samples, which were negative both as a result of visual and $\mathrm{T} / \mathrm{C}$ ratio interpretation but positive according to the PRNT-90, showed a wide range of dilution factors (20 and over 320). Interestingly, the discordant sample with a PRNT-90 titer of $>1: 320$ also showed a relatively low cPass sVNT percentage inhibition value, indicating the possible presence of non-RBD targeting SARS-CoV-2 nAbs in this sample. SARS-CoV-2 nAbs that target proteins other than RBD, such as the N-terminal domain of the $S$ protein, have been isolated in COVID-19 patients [22].

The performance of the cPass sVNT showed high sensitivity and specificity when the PRNT-90 was used as the reference standard. These results are consistent with a previous study that had also performed an evaluation based on the PRNT-90 results [15]. Papenburg et al. also reported perfect sensitivity of the cPass sVNT but various specificities depending on the reference standard used. When compared with the PRNT-50 results, cPass sVNT showed specificities ranging from $91 \%$ to $100 \%$, but, when compared with PRNT-90 results, the specificities ranged from $57 \%$ to $61 \%$. The authors postulated that protocols for the same reference methods could potentially vary across laboratories [23]. The performance of cPass sVNT, especially its specificity, requires further investigation.

Visual interpretation of RDTs is subjective with variable results depending on the interpreter. Analyzing RDTs with SIA has been reported to provide improved and reliable results [18-20]. In this study, when the signal intensity of BZ-nAb was measured using the SIA, the T/C ratio cutoff led to an increase in the sensitivity of the BZ-nAb from $92.5 \%$ to $95.0 \%$, impacting its performance, although at the expense of its specificity. Another advantage of quantifying signal intensity using the $\mathrm{T} / \mathrm{C}$ ratio was that the correlation between the PRNT-90 dilution factor and cPass sVNT percent inhibition value could be analyzed. The BZ-nAb showed a stronger correlation with the cPass sVNT compared to that with the PRNT-90, possibly because of the similarities between the two assays, such as use of purified RBD of S protein and detection of the inhibition of the RBD-ACE2 interaction by $n$ Abs.

This study has limitations. First, a relatively small number of COVID-19 patient sera was evaluated, and longitudinal follow-up could not be carried out due to the lack of availability of samples at different time points. Secondly, the cross-reactivity of BZ-nAb evaluation using the sera containing antibodies against other coronaviruses or syphilis was not performed. Furthermore, while we used the PRNT-90 as the reference, PRNT with other percentages of plaque reduction, such as PRNT-50, were not available for further evaluation. The PRNT is considered the standard method for SARS-CoV-2 nAb detection by many regulatory agencies, but the guidelines do not specify the percentage of plaque reduction that should be evaluated. The more stringent PRNT-90 value selected in the current study for evaluating the diagnostic performances using the COVID-19 patient sera, could had impact in high sensitivity observed in our study. Our results should be interpreted considering that the PRNT-90 has been used as the reference and could 
potentially impact the performance. Future research assessing both PRNT-50 and PRNT90 will be important to examine the BZ-nAb performance for samples with discrepant results between PRNT-50 and PRNT-90 discrepant results, especially PRNT-50 positive and PRNT-90 negative samples.

\section{Conclusions}

Our performance evaluation of the BZ-nAb demonstrated its ability to detect SARSCoV-2 nAbs in COVID-19 patients. The BZ-nAb was found to have a performance comparable to that of the cPass sVNT. An almost perfect agreement was observed between the cPass sVNT and BZ-nAb results. SIA was useful because the application provided improved sensitivity and quantified signal intensity. Measurement of signal intensity of $\mathrm{BZ}-\mathrm{nAb}$ using the $\mathrm{T} / \mathrm{C}$ ratio was associated with an increased sensitivity of BZ-nAb and showed a strong correlation with the PRNT-90 dilution factor and a very strong correlation with cPass sVNT results.

Supplementary Materials: The following are available online at https://www.mdpi.com/article/ 10.3390/diagnostics11122193/s1, Figure S1: BZ-nAb and the T/C ratio measurement using a smartphone-based image analysis application (SIA). Figure S2: Data distribution of PRNT-90 dilution factor, BZ-nAb T/C ratio, and cPass sVNT percent inhibition in PRNT-90-positive and -negative groups.

Author Contributions: Conceptualization, B.K.J. and C.S.L.; Formal analysis, J.Y.; Methodology, J.-Y.B., J.K., M.-S.P., and S.Y.L.; Project administration, C.S.L.; Visualization, J.Y.; Writing—original draft, J.Y.; Writing—review \& editing, J.Y. All authors have read and agreed to the published version of the manuscript.

Funding: This study was supported by a grant of the Korea Health Technology R\&D Project through the Korea Health Industry Development Institute (KHIDI), funded by the Ministry of Health \& Welfare, Republic of Korea (grant number: HR20C0021) and a grant from the Korea university (grant number: K2023141).

Institutional Review Board Statement: The study was conducted according to the guidelines of the Declaration of Helsinki and approved by the Institutional Review Board of Korea University Guro Hospital at 7 April 2021 (2021GR0146).

Informed Consent Statement: Patient consent was waived by the Institutional Review Board of Korea University Guro Hospital as the identities of subjects is completely anonymous and there is minimal risk involved in the study.

Data Availability Statement: Not applicable.

Conflicts of Interest: The authors declare no conflict of interest.

\section{References}

1. Kim, J.H.; Marks, F.; Clemens, J.D. Looking beyond COVID-19 vaccine phase 3 trials. Nat. Med. 2021, 27, 205-211. [CrossRef]

2. Lumley, S.F.; O'Donnell, D.; Stoesser, N.E.; Matthews, P.C.; Howarth, A.; Hatch, S.B.; Marsden, B.D.; Cox, S.; James, T.; Warren, F.; et al. Antibody Status and Incidence of SARS-CoV-2 Infection in Health Care Workers. N. Engl. J. Med. 2021, 384, 533-540. [CrossRef] [PubMed]

3. Piccoli, L.; Park, Y.J.; Tortorici, M.A.; Czudnochowski, N.; Walls, A.C.; Beltramello, M.; Silacci-Fregni, C.; Pinto, D.; Rosen, L.E.; Bowen, J.E.; et al. Mapping Neutralizing and Immunodominant Sites on the SARS-CoV-2 Spike Receptor-Binding Domain by Structure-Guided High-Resolution Serology. Cell 2020, 183, 1024-1042.e21. [CrossRef] [PubMed]

4. GeurtsvanKessel, C.H.; Okba, N.M.A.; Igloi, Z.; Bogers, S.; Embregts, C.W.E.; Laksono, B.M.; Leijten, L.; Rokx, C.; Rijnders, B.; Rahamat-Langendoen, J.; et al. An evaluation of COVID-19 serological assays informs future diagnostics and exposure assessment. Nat. Commun. 2020, 11, 3436. [CrossRef] [PubMed]

5. Muecksch, F.; Wise, H.; Batchelor, B.; Squires, M.; Semple, E.; Richardson, C.; McGuire, J.; Clearly, S.; Furrie, E.; Greig, N.; et al. Longitudinal Serological Analysis and Neutralizing Antibody Levels in Coronavirus Disease 2019 Convalescent Patients. J. Infect. Dis. 2021, 223, 389-398. [CrossRef]

6. Dispinseri, S.; Secchi, M.; Pirillo, M.F.; Tolazzi, M.; Borghi, M.; Brigatti, C.; De Angelis, M.L.; Baratella, M.; Bazzigaluppi, E.; Venturi, G.; et al. Neutralizing antibody responses to SARS-CoV-2 in symptomatic COVID-19 is persistent and critical for survival. Nat. Commun. 2021, 12, 2670. [CrossRef] 
7. Khoury, D.S.; Cromer, D.; Reynaldi, A.; Schlub, T.E.; Wheatley, A.K.; Juno, J.A.; Subbarao, K.; Kent, S.J.; Triccas, J.A.; Davenport, M.P. Neutralizing antibody levels are highly predictive of immune protection from symptomatic SARS-CoV-2 infection. Nat. Med. 2021, 27, 1205-1211. [CrossRef] [PubMed]

8. Walsh, E.E.; Frenck, R.W., Jr.; Falsey, A.R.; Kitchin, N.; Absalon, J.; Gurtman, A.; Lockhart, S.; Neuzil, K.; Mulligan, M.J.; Bailey, R.; et al. Safety and Immunogenicity of Two RNA-Based COVID-19 Vaccine Candidates. N. Engl. J. Med. 2020, 383, 2439-2450. [CrossRef]

9. Jackson, L.A.; Anderson, E.J.; Rouphael, N.G.; Roberts, P.C.; Makhene, M.; Coler, R.N.; McCullough, M.P.; Chappell, J.D.; Denison, M.R.; Stevens, L.J.; et al. An mRNA Vaccine against SARS-CoV-2-Preliminary Report. N. Engl. J. Med. 2020, 383, $1920-1931$. [CrossRef]

10. Folegatti, P.M.; Ewer, K.J.; Aley, P.K.; Angus, B.; Becker, S.; Belij-Rammerstorfer, S.; Bellamy, D.; Bibi, S.; Bittaye, M.; Clutterbuck, E.A.; et al. Safety and immunogenicity of the ChAdOx1 nCoV-19 vaccine against SARS-CoV-2: A preliminary report of a phase 1/2, single-blind, randomised controlled trial. Lancet 2020, 396, 467-478. [CrossRef]

11. Perera, R.A.; Mok, C.K.; Tsang, O.T.; Lv, H.; Ko, R.L.; Wu, N.C.; Yuan, M.; Leung, W.S.; Chan, J.M.; Chik, T.S.; et al. Serological assays for severe acute respiratory syndrome coronavirus 2 (SARS-CoV-2), March 2020. Eurosurveillance 2020, $25,2000421$. [CrossRef] [PubMed]

12. Lau, E.H.Y.; Tsang, O.T.Y.; Hui, D.S.C.; Kwan, M.Y.W.; Chan, W.H.; Chiu, S.S.; Ko, R.L.W.; Chan, K.H.; Cheng, S.M.S.; Perera, R.; et al. Neutralizing antibody titres in SARS-CoV-2 infections. Nat. Commun. 2021, 12, 63. [CrossRef] [PubMed]

13. Nie, J.; Li, Q.; Wu, J.; Zhao, C.; Hao, H.; Liu, H.; Zhang, L.; Nie, L.; Qin, H.; Wang, M.; et al. Establishment and validation of a pseudovirus neutralization assay for SARS-CoV-2. Emerg. Microbes Infect. 2020, 9, 680-686. [CrossRef] [PubMed]

14. Muruato, A.E.; Fontes-Garfias, C.R.; Ren, P.; Garcia-Blanco, M.A.; Menachery, V.D.; Xie, X.; Shi, P.Y. A high-throughput neutralizing antibody assay for COVID-19 diagnosis and vaccine evaluation. Nat. Commun. 2020, 11, 4059. [CrossRef]

15. Taylor, S.C.; Hurst, B.; Charlton, C.L.; Bailey, A.; Kanji, J.N.; McCarthy, M.K.; Morrison, T.E.; Huey, L.; Annen, K.; DomBourian, M.G.; et al. A New SARS-CoV-2 Dual-Purpose Serology Test: Highly Accurate Infection Tracing and Neutralizing Antibody Response Detection. J. Clin. Microbiol. 2021, 59, e02438-20. [CrossRef]

16. Murray, M.J.; McIntosh, M.; Atkinson, C.; Mahungu, T.; Wright, E.; Chatterton, W.; Gandy, M.; Reeves, M.B. Validation of a commercially available indirect assay for SARS-CoV-2 neutralising antibodies using a pseudotyped virus assay. J. Infect. 2021, 82, 170-177. [CrossRef]

17. BioZentech. COVID-19 Neutralizing Ab Test [Instructions for Use]; BioZentech: Seoul, Korea, 2021.

18. Noble, L.; Scott, L.; Stewart-Isherwood, L.; Molifi, S.J.; Sanne, I.; Da Silva, P.; Stevens, W. Continuous quality monitoring in the field: An evaluation of the performance of the Fio Deki Reader ${ }^{\mathrm{TM}}$ for rapid HIV testing in South Africa. BMC Infect. Dis. 2020, 20, 320. [CrossRef]

19. Kalinga, A.K.; Mwanziva, C.; Chiduo, S.; Mswanya, C.; Ishengoma, D.I.; Francis, F.; Temu, L.; Mahikwano, L.; Mgata, S.; Amoo, G.; et al. Comparison of visual and automated Deki Reader interpretation of malaria rapid diagnostic tests in rural Tanzanian military health facilities. Malar. J. 2018, 17, 214. [CrossRef]

20. Visser, T.; Ramachandra, S.; Pothin, E.; Jacobs, J.; Cunningham, J.; Menach, A.L.; Gatton, M.L.; Dos Santos Souza, S.; Nelson, S.; Rooney, L.; et al. A comparative evaluation of mobile medical APPS (MMAS) for reading and interpreting malaria rapid diagnostic tests. Malar. J. 2021, 20, 39. [CrossRef]

21. Kim, Y.J.; Bae, J.Y.; Bae, S.; Hwang, S.; Kwon, K.T.; Chang, H.H.; Lee, W.K.; Cui, C.; Lee, G.E.; Kim, S.W.; et al. Neutralizing Antibody Responses to SARS-CoV-2 in Korean Patients Who Have Recovered from COVID-19. Yonsei Med. J. 2021, 62, 584-592. [CrossRef]

22. Liu, L.; Wang, P.; Nair, M.S.; Yu, J.; Rapp, M.; Wang, Q.; Luo, Y.; Chan, J.F.; Sahi, V.; Figueroa, A.; et al. Potent neutralizing antibodies against multiple epitopes on SARS-CoV-2 spike. Nature 2020, 584, 450-456. [CrossRef] [PubMed]

23. Papenburg, J.; Cheng, M.P.; Corsini, R.; Caya, C.; Mendoza, E.; Manguiat, K.; Lindsay, L.R.; Wood, H.; Drebot, M.A.; Dibernardo, A.; et al. Evaluation of a Commercial Culture-Free Neutralization Antibody Detection Kit for Severe Acute Respiratory Syndrome-Related Coronavirus-2 and Comparison with an Antireceptor-Binding Domain Enzyme-Linked Immunosorbent Assay. Open Forum Infect. Dis. 2021, 8, ofab220. [CrossRef] [PubMed] 